\title{
Adherence of Dentists to the Instructions of Health Regulations during COVID-19 Pandemic
}

\section{Mervat Khoury Absawi, DMD ${ }^{1,2}$, Kholoud Fahoum, BDS ${ }^{1,2^{*}}$, Sarnat Haim, DMD, MSc ${ }^{1}$, Daniel Oren, $D M D^{2,3}$, Fares Kablan, DMD ${ }^{2,3}$ and Samer Srouji, DMD, PhD ${ }^{2,3}$}

${ }^{1}$ Oral and Maxillofacial Surgery, Oral Medicine and Dentistry Institute, Department of Pediatric Dentistry, Galilee Medical Center, Israel

${ }^{2}$ The Azrieli Faculty of Medicine, Bar-llan University, Israel

${ }^{3}$ Oral and Maxillofacial Surgery, Oral Medicine and Dentistry Institute, Galilee Medical Center, Israel

*Corresponding author: Kholoud Fahoum, BDS, Oral and Maxillofacial Surgery, Oral Medicine and Dentistry Institute, Department of Pediatric Dentistry, Galilee Medical Center, Nahariya, Israel; The Azrieli Faculty of Medicine, Bar-llan University, Safed, Israel

\begin{abstract}
Background: A large percentage of medical personnel including dentists, acquired COVID-19 while treating patients. Worldwide health regulatory authorities have devised and distributed guidelines and information for dental staff, to prevent transmission of the virus. This study aimed to assess the degree of dentist adherence to these recommendations during the COVID-19 pandemic.
\end{abstract}

Methods: An online questionnaire was distributed via social media among dental practitioners in Israel who worked during the COVID-19 outbreak.

Results: The survey was completed by 144 dentists; it was found that dentist adherence to all of the recommendations was $69.83 \%$. Many dentists $(46.78 \%$ ) reported on use of extra protection which were not required, with $36.8 \%$ using N95 face masks. knowledge of COVID-19 was rated as "very good" by $37.1 \%$ of responders. However, only $25.5 \%$ felt "highly protected" by personal protective equipment.

Conclusion: Stricter regimens should be applied for dealing with this challenging virus. Specific strategies should be followed to ensure good practice in order to improve the safety of dentists and patients.

\section{Keywords}

Personal protective equipment, Coronavirus disease 2019, Aerosol, N95

\section{Abbreviations}

MOH: Ministry of Health; COVID-19: Coronavirus Disease
2019; PPE: Personal Protective Equipment; ACE2: Angiotensin-Converting Enzyme-2; SARS-CoV-2 virus: Severe Acute Respiratory Syndrome Coronavirus 2; CDC: Centers for Disease Control and Prevention; FFRs: Filtering Facepiece Respirators; ADA: American Dental Association; WHO: World Health Organization

\section{Background}

Many medical personnel acquired severe acute respiratory syndrome coronavirus 2 (SARS-CoV-2 virus) while treating patients [1]. Dentists were no exception, due to their proximity to patient and the use of devices prone to produce spraying $[2,3]$. In light of this high occupational risk, dentists should consider every patient as infected [4], and should be aware of preventive strategies to protect themselves as well as their patients. The worldwide health regulatory authorities, such as the Centers for Disease Control (CDC) [5] and the American Dental Association (ADA) [6], devised and distributed new guidelines and information for dental staff to prevent transmission of the SARS-CoV-2 virus. These include strict adherence to modified personal protective equipment (PPE) guidelines, including use of impermeable gowns, goggles and/or face shields and respirators/surgical masks, in addition to standard precautions and patient management. 
The $\mathrm{N} 95$ mask is one of several types of respirators which belongs to filtering facepiece respirators (FFRs). Since aerosol particle size is less than $50 \mu \mathrm{m}$ which can stay airborne for a time, N95 is characterized by its $95 \%$ filtration ability of small particles of up to $0.3 \mu \mathrm{m}$. The mask comes in several shapes, tightly fits the face, providing a good seal around the mouth and nose, and resists fluid penetration. The N95 also comes in different designs and models, which should be judiciously selected, depending on the work conditions and specific purpose. For example, a N95 mask with exhalation valves should not be used during sterile procedures [7].

Goggles are worn to protect the eyes from infected aerosol $[8,9]$, and are especially important during the current COVID-19 pandemic, owing to the connection between the conjunctival mucosa and upper respiratory tract by the nasolacrimal duct, where they share the same receptor; angiotensin-converting enzyme-2 (ACE2), through which the SARS-CoV-2 virus gains entry to the cell [9]. Face shields provide better and broader protection, with less fogging than goggles [8].

Latex gloves are more resistant than latex-free gloves, but there are several factors that reduce their integrity by causing micro perforations, such as; > 60 minutes of usage, washing with soap, alcohol or chlorhexidine, and putting on without permitting total drying of the sterilizing alcoholic materials applied on the hands [8]. Gowns, which are available in different types, offering varying levels of protection, should also be worn and appropriately chosen [8].

SARS-CoV-2 virus persist in aerosol for up to 3 hours as reported by van Doremalen, et al. [10] and the air in the operating room remains contaminated for several hours which places the dental staff at risk after removing of the PPE, as well as the subsequent patients [8].

The aim of this cross-sectional study was to assess the adherence level of dentists to the infection prevention control precautions and to PPE recommendations during the COVID-19 pandemic, to identify factors impacting adherence and inquire about their sense of security.

\section{Methods}

The survey was conducted in Israel between March 17, 2020 and April 30, 2020. At the time were the Israeli Ministry of Health limited dental treatments to urgent dental care only.

Research ethics: Written consent was obtained from the Institutional Review Board for clinical trials (IRB-Helsinki committee) at the Galilee Medical Center, Nahariya, Israel. NHR-0083-20.

Consent was automatically obtained by the responders at the beginning of the questionnaire by the statement: Whenever you fulfill the questionnaire it means that you automatically provided your consent to be part of the research.
The research was carried out by circulating an online anonymous Questionnaire via Facebook (Facebook Inc. California, USA) and WhatsApp ${ }^{\odot}$ (WhatsApp Inc. California, USA), among dentists who worked during the onset of COVID-19 outbreak. The questionnaire formulation and clarity of the questions, which were based on the recommendations of the CDC [5], were validated based on colleagues' and statistician's opinion. It was designed in Hebrew and consisted of 29 questions about dentists' demographic characteristics, their attitude toward treatment, infection control and adherence to PPE. Most questions where dichotomous, while some had four rank values (not at all, mildly, moderately, and highly).

The adherence score was computed as the proportion of the guidelines that were implemented by responders, out of the total recommended "Guidelines of the Worldwide Health Regulations. "Extra protection and precautions" adherence score was computed in a similar manner and total adherence score was computed based on both; "Guidelines of the Worldwide Health Regulations" and "Extra protection and precautions".

\section{Statistical Analysis}

Quantitative data are presented as mean and standard deviation (SD). Qualitative data are presented with frequencies and percentages. Quantitative data were compared between groups using the T-test. Ordinal data were compared with the Wilcoxon Test. Results were considered significant if $P<0.05$. The analysis was performed using IBM SPSS Statistics software, version 25.

\section{Results}

In total, 156 dentists responded to the survey, of whom 144 answered most of the questions, the total responsiveness rate was $92 \%$.

The vast majority of responders were general practitioners $(79.9 \%)$, and the rest were specialists or residents in various dental specialties, $52.4 \%$ were men, and $53.8 \%$ were between $25-34$ years of age. Most $(69.83 \%)$ dentists adhered to the worldwide health regulations, with $92.6 \%$ reporting on use of surgical masks, $78 \%$ reporting on use of face shield/goggles, $67.4 \%$ reporting on use of gown, and $36.8 \%$ reporting on use of $\mathrm{N} 95$ masks. Sterilization of nitrous oxide parts was performed by $88.4 \%$ and $24.8 \%$ of responders reported on increased frequency of rubber dam usage. Most dentists $(63.9 \%)$ avoided providing aerosol-producing treatments, $51.1 \%$ of dentists measured the patient's temperature on arrival, and $88.7 \%$ always asked initial screening questions (Table 1).

Adherence scores were not significantly impacted by professional seniority level; specialists; $74.83 \%$, resident dentists; $71.59 \%$, general practitioners; $68.87 \%$.

Extra protection and precautions which were not 
Table 1: Dentists adherence to the international health regulations (Ex: CDC, ADA).

\begin{tabular}{|l|l|}
\hline & $\begin{array}{l}\text { Responders } \\
\text { who answered } \\
\text { YES } \\
\text { N (\%) }\end{array}$ \\
\hline Use surgical mask & $126(92.6 \%)$ \\
\hline Use N95 & $50(36.8 \%)$ \\
\hline Wear gowns & $91(67.4 \%)$ \\
\hline Use face shield/goggles & $106(78 \%)$ \\
\hline Sterilization of $\mathrm{N}_{2} \mathrm{O}$ parts & $114(88.4 \%)$ \\
\hline Increased frequency of rubber dam usage & $31(24.8 \%)$ \\
\hline Avoid aerosol treatments & $78(63.9 \%)$ \\
\hline $\begin{array}{l}\text { Patient temperature measurement before } \\
\text { treatment }\end{array}$ & $68(51.1 \%)$ \\
\hline Ask initial screening questions & $118(88.7 \%)$ \\
\hline Total score of full adherence, mean \pm SD & $69.83 \pm 20.24$ \\
\hline
\end{tabular}

Table 2: Most frequent dental emergencies and patient management.

\section{n (\%)}

\section{Most frequent emergency cases:}

\begin{tabular}{|l|l|}
\hline Spontaneous pain & $97(75.8 \%)$ \\
\hline External/internal swelling & $14(10.9 \%)$ \\
\hline Pain during eating & $8(6.3 \%)$ \\
\hline $\begin{array}{l}\text { Other (ex: dental trauma, hyper-sensitivity, } \\
\text { tooth mobility) }\end{array}$ & $9(7 \%)$ \\
\hline
\end{tabular}

\section{Most frequent treatments}

Pulpotomy/extirpation $66(53.2 \%)$

Extractions $27(21.8 \%)$

Temporary restorations $16(12.9 \%)$

Prescribing antibiotics $15(12.1 \%)$

Table 3: Self-perceived level of protection and knowledge of COVID-19 among dentists.

$$
\text { n (\%) }
$$

How do you estimate your knowledge of COVID-19

\begin{tabular}{|l|l|}
\hline Good enough & $88(62.9 \%)$ \\
\hline Very good & $52(37.1 \%)$ \\
\hline Level of sense of protection by the PPE: & \\
\hline Not at all & $15(10.9 \%)$ \\
\hline Mildly protected & $38(27.7 \%)$ \\
\hline Moderately protected & $49(35.8 \%)$ \\
\hline Highly protected & $35(25.5 \%)$ \\
\hline
\end{tabular}

required by the worldwide health regulations, were implemented by $46.78 \%$ of dentists, including use of double gloves (45.2\%) and/or a medical cap (49.2\%), increased frequency of face mask change (67.7\%), and request from patients to rinse with hydrogen peroxide $1 \%$ before treatment $(24.8 \%)$, because of the vulnerability of the virus to oxidation [11].

Most dentists (75.8\%) reported that the main patient complaint was spontaneous pain and the most common treatment was pulpotomy/pulp extirpation (Table 2).

Knowledge of COVID-19 was ranked "good enough" by $62.9 \%$ and "very good" by $37.1 \%$ of the responders. A larger percentage of dentists who reported on "very good" knowledge of COVID-19 adhered to the worldwide health regulations in addition to using of extra protection and precautions than those who reported "good enough" knowledge, $(68.0 \pm 20.2$ and $59.2 \pm 20.3$, respectively; Figure $1 ; p=0.014)$. Most responders felt moderately $(35.8 \%), 25.5 \%$ felt highly protected, while $27.7 \%$ felt mildly protected and $10.9 \%$ did not feel protected at all (Table 3). A larger percentage of dentists who used a N95 mask felt "highly" protected by the PPE than dentists who did not use them (36\% and $19.8 \%$, respectively; $p=0.013$; Figure 2 ), the same for dentists who used a face shield and/or goggles (32.1\% and 3.3\% respectively; $p=0.001$ ), and fewer N95 mask users felt "not protected at all" as compared to nonusers ( $4 \%$ and $15.1 \%$, respectively; $p=0.014$ ).

\section{Discussion}

Continued dental services have been essential throughout the COVID-19 pandemic. In order to operate safely and efficiently, dentists have been urged to follow the worldwide regulatory guidelines adapted to address the threats of the current health crisis. This study provided insights into dentist adherence to the new guidelines relating to PPE usage, and standard precautions, including patient management. Overall, $69.83 \%$ of the survey responders reported on full adherence to the recommendations.

Knowledge of COVID-19 mechanisms of action and mode of transmission enhances combat efforts. In this study, a direct relation was found between responder knowledge level of COVID-19 and adherence to the international recommendations and implementation of extra protective measures during treatment even when they were not required. Even though there was no statistical difference between professional seniority and adherence scores, specialists adhered more than other dentists, likely because of their higher academic level.

Good prevention and protection practices are preferred over treatment of the disease, and proper use of PPE is the key to success in preventing contamination. However, although the protection factor of N95 respirators against viral aerosols was demonstrated to be 30 times greater than normal surgical masks [7], the Israeli Ministry of Health, unlike the CDC and WHO $[5,12,13]$, did not obligate their use or the use of equivalent or higher level respirators in aerosolgenerating procedures. Most dentists responding to this survey used surgical masks which are not indicated for aerosol-producing treatments that contain droplets with high virulent pathogens [8] because they lack the ability of sealing and filtering small particles [9]. 


\section{Level of knowledge vs Level of adherence (1)}

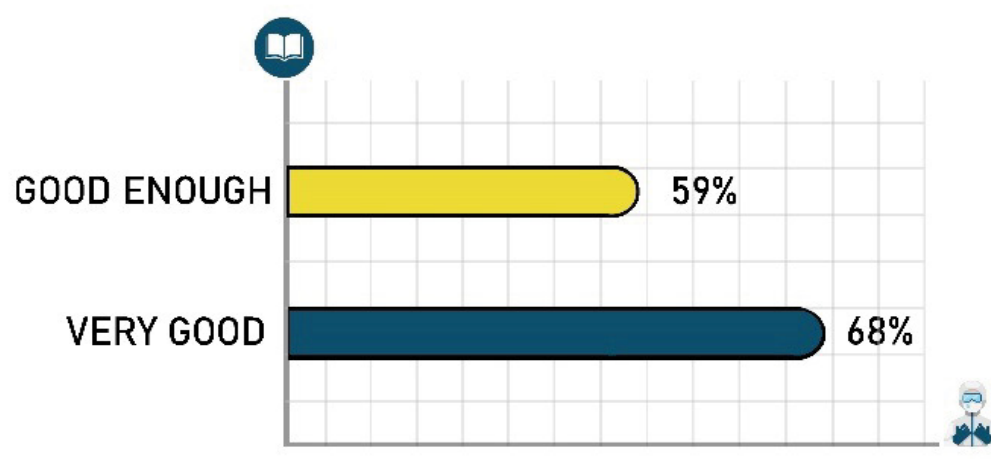

$P=0.014$

Figure 1: Level of COVID-19 knowledge vs. level of guidelines adherence.

Level of COVID-19 knowledge vs. level of guidelines adherence. Dentists who reported on "very good" knowledge of COVID-19 adhered more than those who reported "good enough" knowledge $(p=0.014)$.

\section{Self-perceived highly protected \\ VS \\ Using N95 mask}

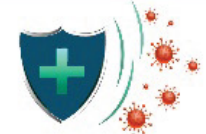

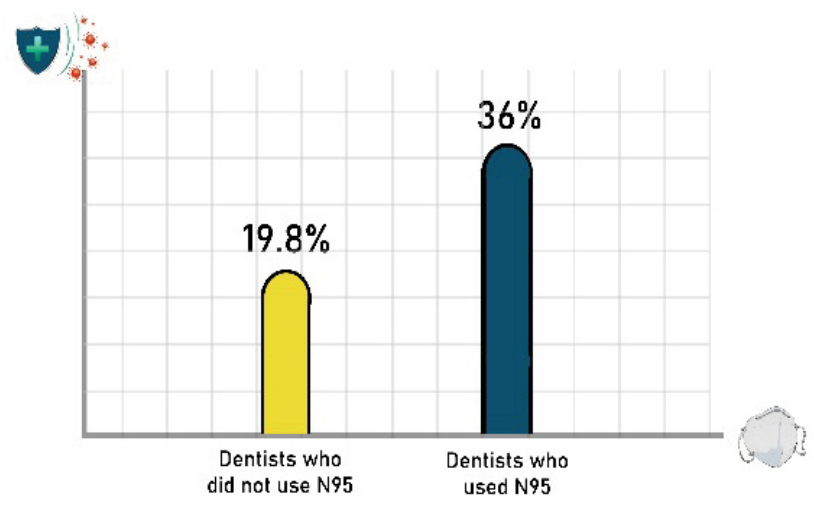

$P=0.013$

Figure 2: Self-perceived high protection as a function of N95 mask usage.

Self-perceived high protection as a function of N95 mask usage. Dentists who used N95 mask felt more "highly protected" than those who did not use them ( $36 \%$ and $19.8 \%$, respectively; $p=0.013$ ).

A high percentage of dentists did not feel highly protected by the PPE (74.5\%), but dentists who used N95 mask and face shields and/or goggles, felt significantly more highly protected than dentists who did not use them. Sense of self-protection is an important factor in confidently providing the right treatment without stress.

It is important to note that although contamination can arise from improper PPE donning and doffing sequences, to date, there are no universally accepted protocols for these activities [7]. This underscores the need for well-defined guidelines regarding sequence and location of PPE donning and doffing to prevent contamination.

Although most dentists reportedly tried to avoid aerosol-producing treatments (63.9\%), pulp extirpation was the most frequently performed emergency procedure, likely due to high incidence of spontaneous pain cases, as it is not always useful to prescribe an antibiotic or analgesic. Health regulations should regularly update and direct dentists to use alternative safe and non-aerosol-producing treatment modes, such 
as use of conventional files for root canal treatments, rather than rotary instruments, tooth decay excavation in place of drilling [9], and use of materials that facilitate caries removal.

In addition, dentists should be familiar with the analgesic drugs that should be avoided, e.g., ibuprofen, due to their enhancing effect on ACE2 expression, which can increase susceptibility to SARS-CoV-2. Moreover, to be familiar with oral complications such as ulcers, lichenoid reaction and melanotic pigmentation of the oral mucosa, that can be induced by medications prescribed for COVID-19 symptoms, such as, chloroquine, as well as with the clinical manifestations of COVID-19 that affect the orofacial region like dysgeusia and burning mouth syndrome [9].

\section{Conclusions}

Although SARS-CoV-2 vaccines are being slowly rolled out, the COVID-19 pandemic is far from over. To improve and ensure a higher percentage of adherence to worldwide health regulations, it is important to activate an updating process, that aims to disseminate information regarding the disease among dental teams, and to ensure best practices and management approaches in order to face this and upcoming challenging viruses without fear of infection.

More specifically, we suggest establishing:

1. Educational interventions by the Israeli health regulatory bodies to increase dentist awareness and knowledge regarding COVID-19.

2. Mandatory PPE equipment and training courses to reduce errors.

3. Updated guidelines, including mandatory usage of N95 face masks and minimum 30 minutes intervals between patients to allow for proper operating room ventilation.

4. A process to regularly update guidelines as our understanding of disease prevention and treatment evolves.

\section{References}

1. Khader $Y, A$ I Nsour M, Al-Batayneh OB, Saadeh R, Bashier $\mathrm{H}$, et al. (2020) Dentists' awareness, perception, and attitude regarding COVID-19 and infection control: Crosssectional study among jordanian dentists. JMIR public Heal Surveill 6: e18798.

2. Meng L, Hua F, Bian Z (2000) Coronavirus Disease 2019 (COVID-19): Emerging and future challenges for dental and oral medicine. J Dent Res 99: 481-487.

3. Spagnuolo G, De Vito D, Rengo S, Tatullo M (2020) COVID-19 outbreak: An overview on dentistry. Int J Environ Res Public Health 17: 2094.

4. Mallineni SK, Innes NP, Raggio DP, Araujo MP, Robertson MD, et al. (2020) Coronavirus disease (COVID-19): Characteristics in children and considerations for dentists providing their care. Int J Paediatr Dent 30: 245-250.

5. Guidance for dental settings.

6. CDC guidance for dental settings echoes ADA guidance.

7. Umer F, Haji Z, Zafar K (2020) Role of respirators in controlling the spread of novel coronavirus (COVID-19) amongst dental healthcare providers: A review. Int Endod J 53: 1062-1267.

8. Bizzoca ME, Campisi G, Lo ML (2020) Covid-19 Pandemic: What changes for dentists and oral medicine experts? A narrative review and novel approaches to infection containment. Int J Environ Res Public Health 17: 3793.

9. Odeh ND, Babkair H, Abu-Hammad S, Borzangy S, AbuHammad A, et al. (2020) COVID-19: Present and future challenges for dental practice. Int $\mathrm{J}$ Environ Res Public Health 17: 3151.

10. Wesemann C, Pieralli S, Fretwurst T, Nold J, Nelson K, et al. (2020) 3-D Printed protective equipment during covid-19 pandemic. Materials 13: 1997.

11. Peng X, Xu X, Li Y, Cheng L, Zhou X, et al. (2020) Transmission routes of 2019-nCoV and controls in dental practice. IJOS.

12. Rational use of personal protective equipment (PPE) for coronavirus disease (COVID-19) and considerations during severe shortages. WHO.

13. Mask use in the context of COVID-19. 